\title{
Generic framework for vessel detection and tracking based on distributed marine radar image data
}

\author{
Gregor Siegert $^{1}$ (D) Julian Hoth ${ }^{1} \cdot$ Paweł Banyś $^{1} \cdot$ Frank Heymann $^{1}$
}

Received: 29 March 2017 / Revised: 8 June 2017 / Accepted: 18 April 2018

(c) CEAS 2018

\begin{abstract}
Situation awareness is understood as a key requirement for safe and secure shipping at sea. The primary sensor for maritime situation assessment is still the radar, with the AIS being introduced as supplemental service only. In this article, we present a framework to assess the current situation picture based on marine radar image processing. Essentially, the framework comprises a centralized IMM-JPDA multi-target tracker in combination with a fully automated scheme for track management, i.e., target acquisition and track depletion. This tracker is conditioned on measurements extracted from radar images. To gain a more robust and complete situation picture, we are exploiting the aspect angle diversity of multiple marine radars, by fusing them a priori to the tracking process. Due to the generic structure of the proposed framework, different techniques for radar image processing can be implemented and compared, namely the BLOB detector and SExtractor. The overall framework performance in terms of multi-target state estimation will be compared for both methods based on a dedicated measurement campaign in the Baltic Sea with multiple static and mobile targets given.
\end{abstract}

Keywords Maritime situation awareness $\cdot$ Multi-target tracking $\cdot$ Multi-radar fusion $\cdot$ IMM-JPDA filtering $\cdot$ Radar image processing $\cdot$ BLOB detector $\cdot$ SExtractor

\section{Introduction}

The maritime domain is facing several challenges that call for robust and reliable schemes of situation awareness. Besides maritime transport being considered as backbone of globalization and enabler of international trade [41], a clear trend can be witnessed in research and industry towards autonomy in maritime infrastructures and shipping $[9,26]$ placing high demands on security and safety. The radically different concept of autonomous vessels compared to nowadays maritime navigation will certainly call for more sensors, more data and more interaction between different actors. In general, we can separate surveillance from navigation-driven demands on the maritime situation picture. The former calls for a maximized ship detection rate, while the latter requires the localization of objects to be as accurate as possible for collision avoidance. A promising approach for

Gregor Siegert

gregor.siegert@dlr.de

1 German Aerospace Center (DLR), Institute of Communications and Navigation, Kalkhorstweg 53, 17235 Neustrelitz, Germany increasing both, detection rate and accuracy of localization, is to perform data fusion within a distributed network of sensors. With this approach, we do not only extend the area of coverage but also benefit from diverse aspect angles in the overlapping areas. Applying the idea of distributed sensors to the maritime domain clearly defines the main contributors to such a network. As sensor we consider the radar, which is still the primary sensor for collision avoidance and situation assessment to this day [19]. In fact, vessels of $300 \mathrm{gt}$ and above are required to operate at least an X-Band radar on-board. Due to this fact, we can assume a spatial distribution and diversity in terms of aspect angles between different radar platforms, i.e., vessels at sea. In this work we present a framework for situation assessment based on multiple marine radars. The framework comprises a multi-target tracker, namely an Interacting Multiple Model (IMM)-Joint Probabilistic Data Association (JPDA) filter, which is conditioned on fused radar measurements. Target acquisition and depletion is achieved automatically. In contrast to classical radar signal processing (as described in [8]), we propose the use of image processing techniques for target detection from the displayed radar images. Several advantages can be identified with this approach: not only that the majority of 
commercial marine radar systems becomes accessible via their video interface. It also allows for applying computer vision algorithms for additional feature extraction from objects seen in the radar [18]. In this work, we compare the performance in multi-target state estimation for two different image-based target detectors: the standard BLOB detector from the OpenCV library as well as the Source Extractor (SExtractor) [3], known from astronomy for star detection. Based on measurement data from a multi-target, multi-radar campaign in the Baltic Sea we will demonstrate and analyze the working principle of the proposed scheme for both image-based target detectors.

The remainder of this document is organized as follows. The related work is described in Sect. 2. The framework for situation assessment including both proposed methods for target detection in marine radar images is presented in Sect. 3. Both methods will be evaluated and compared in their performance in Sect. 4 based on measurement data. A conclusion and outlook is given in Sect. 5 .

\section{Related work}

The topic of maritime situation awareness is of great interest to the community, which has led to a variety of multisensor, multi-target tracking (MTT)-related publications in the literature. From the application side, anomaly detection is the driving force for many contributions, such as [32] and [33], in which nominal traffic patterns are extracted from historical Automatic Identification System (AIS) data. Based on the individual models that represent this ground-truth the behavior of monitored vessels can be evaluated. This, however, assumes a solid knowledge about the current traffic situation. Thus, the refinement of the situation picture is the motivation for many works applying methods of multisensor, multi-target tracking. In [16] and [30] AIS data are fused with Synthetic Aperture Radar (SAR) imagery and/ or coastal radar to provide a reliable and precise situation picture for large-scale maritime surveillance. In the same context different authors, such as [7,29] and [31], have also studied the benefits of fusing multiple coastal Over-theHorizon (OTH) radars in terms of an improved MTT performance and increased area of coverage. As opposed to shorebased surveillance, on-board maritime situation assessment was also covered in [23] providing an overview of different techniques for fusing radar with AIS. In [37] and [39], implementations of IMM-Multi-Sensor PDA (MSPDA) and IMM-JPDA filters were applied to fuse radar and AIS measurement data in a single- and multi-target environment, respectively. It was shown that due to the complementary nature of both sensors, the overall completeness of the monitored area can be increased by compensating the outages of one or the other. The next step in this development is to incorporate multiple radars located not at the coast but at spatially distributed vessels. By exploiting the aspect angle diversity it is expected to increase the accuracy for tracked targets in the overlapping regions of both radars. The general benefits from multi-radar fusion are well described in [42] and applied to the maritime domain in [10].

\section{Generic framework for maritime situation assessment}

The main scope of this work is the introduction of a generic framework for maritime situation assessment. In principle, this framework allows for fully automated multi-target acquisition and tracking based on multi-radar fusion. For the latter, we propose the use of radar image-based processing techniques for target candidate extraction. Figure 1 depicts the modular structure of the framework identifying the individual processing stages that will be described in more detail in this section.

\subsection{Target candidate extraction}

To update the IMM-JPDA filter with measurements, target candidates need to be detected and extracted from radar first. As is depicted in Fig. 1, the approach to extract radar target information is based on image processing rather than working directly in the radar signal domain. While this may introduce additional error sources that origin from mapping the radar target data from signal to image domain it also yields some clear advantages. First of all, the vast variety of known image processing techniques becomes available; not only to extract point sources but also the shape and dimensions of potential targets, which could be exploited in future work. Second, practical experience shows that most commercial marine radar systems offer at least a video output as interface. Working with the images allows the integration of nearly all available radar systems. It has to be stated, though, that the overall design of the framework is not limited to radar image processing methods. In fact, the multi-target tracker (IMM-JPDA filter in Fig. 1) expects radar target measurements in polar domain, i.e., in range and bearing. In this way, we are flexible enough to process measurements obtained directly from the original radar azimuth scans. For this work, however, we propose the use of two different computer vision techniques for target candidate extraction from radar images. To extract target candidates from the current radar image at time $k$, the following generic procedure is applied:

1. Masking the image to eliminate features of the user interface, e.g., colored heading lines, blob in center, radar information tables. 


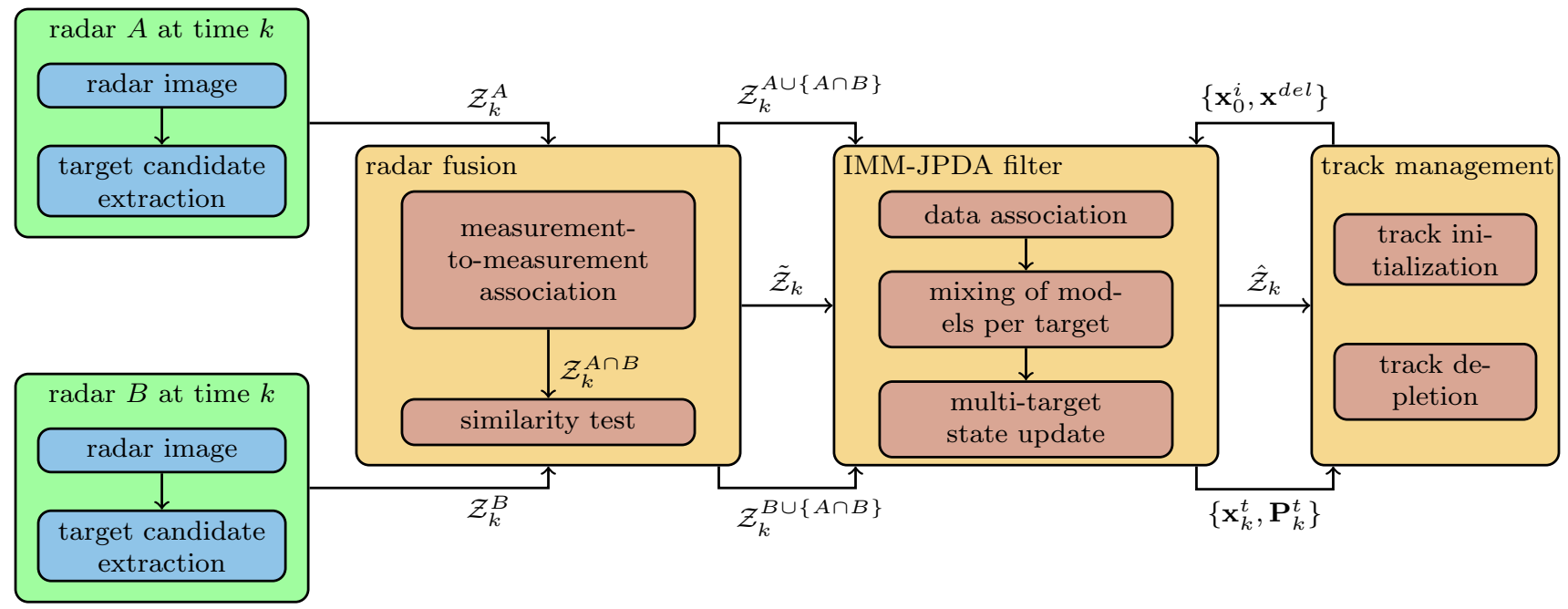

Fig. 1 Block diagram of the proposed system architecture; at time $k$ from each of the radars $A$ and $B$ a new radar image is captured. From these images a set of measurements $\mathcal{Z}_{k}^{A}$ and $\mathcal{Z}_{k}^{B}$ is extracted. The measurements that can be associated to each other constitute the subset $\mathcal{Z}_{k}^{A \cap B}$. The associated pairs within this set are fused yielding $\tilde{\mathcal{Z}}_{k}$, which is used as input to the multi-target tracker. The remaining measurements from radar $A$ and $B$ are captured in $\mathcal{Z}_{k}^{A \cup\{A \cap B\}}$ and $\mathcal{Z}_{k}^{B \cup\{A \cap B\}}$, respectively, and are directly used as measurement input

2. Conversion of the image from RGB to gray-scale (weighted average from color channels) and intensity thresholding.

3. Application of either BLOB detection or SExtractor. Both return the center of mass for each detected target as pixel coordinates.

4. These pixel coordinates are converted to range and bearing relative to the position of the vessel carrying the radar.

The set of extracted target measurements from the current image of radar $s \in\{A, B\}$ is defined as $\mathcal{Z}_{k}^{s}=\left\{\mathbf{z}_{k}^{s, 1}, \ldots, \mathbf{z}_{k}^{s, M}\right\}$, with the $j$ th measurement vector $\mathbf{z}_{k}^{s, j}=\left[z_{k}^{r}, z_{k}^{b}\right]^{\mathrm{T}}$ comprising range and bearing of the target candidate.

\subsubsection{The BLOB detector}

In the literature, various approaches are known to detect low-level objects (i.e., blobs) from image data sets that find application in many image-based classification or tracking problems, such as in [17] or [20]. The BLOB detector used in this work is an implementation provided by the OpenCV framework $^{1}$. In this case, blobs are grouped and merged within several binary sub-images that are formed from initial intensity threshold settings. These blobs are then filtered

\footnotetext{
${ }^{1}$ OpenCV 3.1.0: https://github.com/Itseez/opencv.git.
}

to the IMM-JPDA filter. This multi-target tracker computes the joint data association probabilities and performs the multi-target state update by conditioning and mixing a set of dynamic models per target. The state estimate for the $t$ th target at time $k$ is then $\left\{\mathbf{x}_{k}^{t}, \mathbf{P}_{k}^{t}\right\}$. This set, together with all measurements $\hat{\mathcal{Z}}_{k}$ that could not be associated to any of the existing targets are forwarded to the track management. Here, tracks may be initialized $\left\{\mathbf{x}_{0}^{i}\right\}$ or deleted $\left\{\mathbf{x}^{d e l}\right\}$

based on features such as convexity, circularity, inertia, size and intensity. Figure $2 \mathrm{a}-\mathrm{c}$ shows the radar processing stages, from the original, to the masked and finally to the detected blobs.

\subsubsection{The SExtractor}

To demonstrate the flexibility of the proposed framework, a second algorithm for target candidate detection and extraction was adopted, which is originally known from astronomy as SExtractor [3]. The SExtractor is specifically designed to process large digital images to detect stars, galaxies and other astronomical objects. Special care is taken to achieve speed and robustness of the object detection regardless of the objects' shape and size [3]. Although the SExtractor is designed for astronomical images it can also be used to extract objects in radar images as the objects are similar from a computer vision point of view. It has for instance been applied in [18] showing its advantage over Automatic Radar Plotting Aid (ARPA)-based localization of objects in radar. The SExtractor uses the following six steps for the analysis of an image:

1. Background estimation,

2. Thresholding,

3. Deblending,

4. Detections filtering,

5. Photometry, 


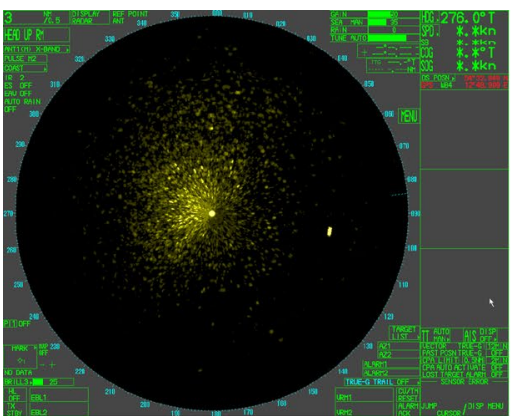

(a)

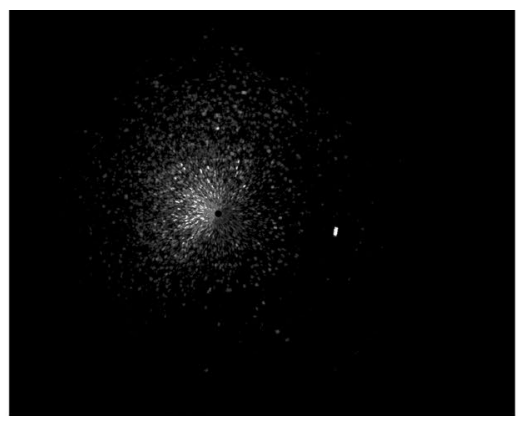

(b)

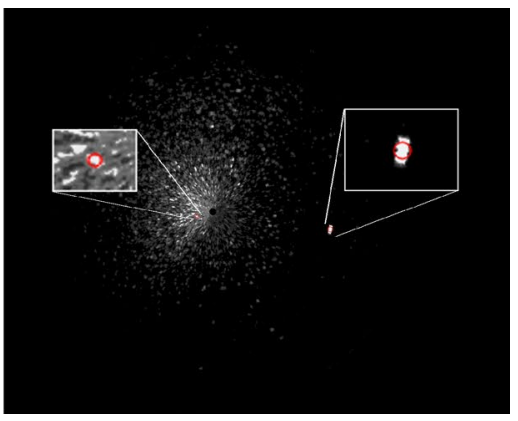

(c)

Fig. 2 Processing chain for one radar image at time $k$ to extract the target candidates. a Original radar image. b Image after background subtraction and gray-scale conversion. $\mathbf{c}$ Extracted target candidates (red circles) at time $k$ after blob detection

\section{Star/galaxy separation.}

Background estimation is done by $\kappa \cdot \sigma$-clipping and mode estimation [12]. Thresholding is applied using Lutz's onepass algorithm [27]. This is followed by deblending of merged objects since objects close to each other may have been extracted as single objects. Hereby, the distribution of the signal strength within the object is described through a tree structure to decide whether a separation in multiple objects is warranted [3]. The detections are then filtered to remove noise near real objects using a First-In First-Out (FIFO) stack. The last two steps are not required in radar images as these are specific to astronomical images and, therefore, not implemented.

\subsection{Radar measurement-to-measurement association}

Before fusing radar target measurements from different sources they need to be associated (see Fig. 1). This becomes evident considering a single radar scan, which typically contains not one but a number of detections. In this study, a two-step procedure to find the correct measurement-tomeasurement associations is used.

1. First, $N_{A}$ measurements from radar $A$ need to be paired with $N_{B}$ measurements from radar $B$. The Global Nearest Neighbor (GNN) rule is applied, yielding bijective associations based on the inter-measurement distance. implementation to find the correct, cost-optimized pairing.

2. Second, each associated pair of measurements needs to pass an additional hypothesis test before fusion:

$H_{0}$ : both measurements origin from the same target
$H_{1}$ : measurements origin from different targets/clutter

For this purpose, we exploit the approximate model of a radar target candidate's position by its mean and its corresponding covariance. This allows the computation of the Bhattacharyya similarity measure [4] between the two distributions describing the same target from radar $A$ and radar $B$. Only if the Bhattacharyya coefficient exceeds a certain threshold $h_{B}, H_{0}$ is accepted and both measurements are fused according to the scheme we will describe in Sect. 3.3.

The Bhattacharyya measure computes the distance $D_{B}$ between two distributions, which is closely linked to the Bhattacharyya coefficient $B C$. The latter expresses the amount of overlap between both distributions. In case of two normal distributions $p$ and $q$, these measures are defined as

$D_{B}(p, q)=\frac{1}{8}\left(\boldsymbol{\mu}_{p}-\boldsymbol{\mu}_{q}\right)^{\mathrm{T}} \boldsymbol{\Sigma}^{-1}\left(\boldsymbol{\mu}_{p}-\boldsymbol{\mu}_{q}\right)+\frac{1}{2} \ln \left(\frac{\operatorname{det} \boldsymbol{\Sigma}}{\sqrt{\operatorname{det} \boldsymbol{\Sigma}_{p} \operatorname{det} \boldsymbol{\Sigma}_{q}}}\right)$,

with

$\boldsymbol{\Sigma}=\frac{\boldsymbol{\Sigma}_{p}+\boldsymbol{\Sigma}_{q}}{2}$

and

$D_{B}(p, q)=-\ln (B C(p, q))$.

\subsection{Radar measurement fusion}

The next step in the processing chain of Fig. 1 addresses the fusion of extracted target candidates. Different strategies for fusing data from multiple and distributed radars can be distinguished. Four general configurations were defined in [13] for data fusion in a sensor network. In this study, we 


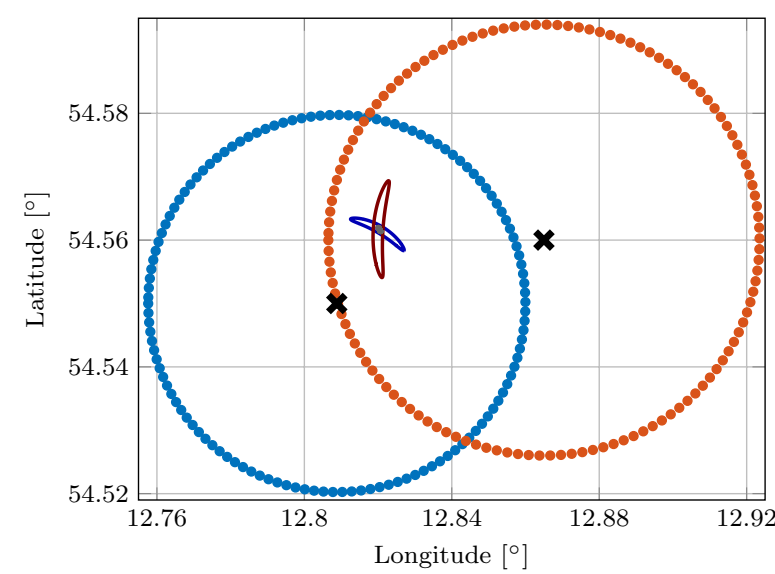

(a)

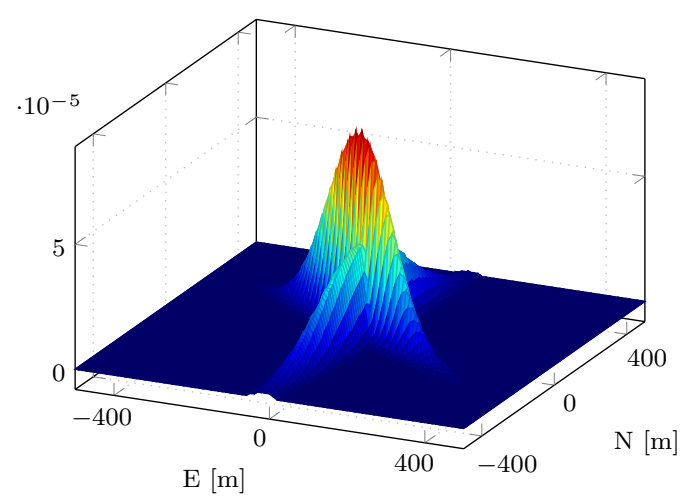

(c)

Fig. 3 Sensor fusion by merging two radar target detections. Each target measurement is approximated as Gaussian, being described via its mean and its covariance. a Two radar systems at different locations (black crosses), with different ranges (blue and red dotted circles). A target (gray blob) is located within the overlapping area and detected by both radars. The measurement uncertainty is projected as banana-

focus on a type III configuration, in which a measurementto-measurement association and fusion rule is applied prior to the actual tracking. As can be seen in the flow chart of Fig. 1, two sets of target candidate measurements $\mathcal{Z}_{k}^{A}$ and $\mathcal{Z}_{k}^{B}$ are generated from both radars. The radar fusion process yields a new set $\tilde{\mathcal{Z}}_{k}$, in which each target measurement is described via its mean and the corresponding sensor noise covariance $\left\{\tilde{\mathbf{z}}_{k}^{t}, \tilde{\mathbf{R}}_{k}^{t}\right\}$. The methodology for radar measurement fusion can be described as follows. Consider a single vessel located within the overlapping area of two marine radars as is shown in Fig. 3. Under the assumption that radar $s \in\{A, B\}$ has detected the target, a sensor measurement $\mathbf{z}_{k}^{s}$ is obtained that represents the true target position with a certain probability following the distribution of

$p\left(\mathbf{z}_{k}^{s}\right) \sim \mathcal{N}\left(\boldsymbol{\mu}=\mathbf{z}_{k}^{s}, \mathbf{\Sigma}=\mathbf{R}_{k}^{s}\right)$

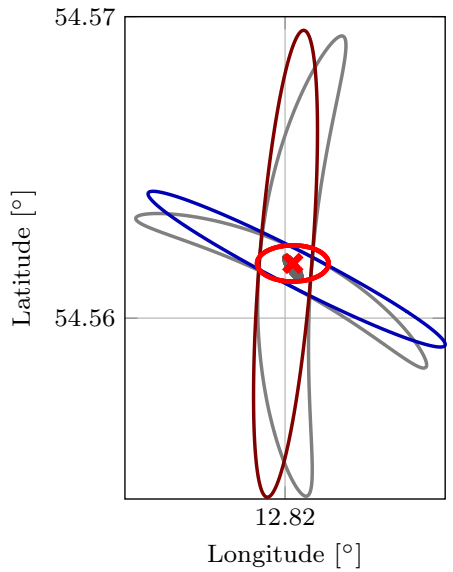

(b)

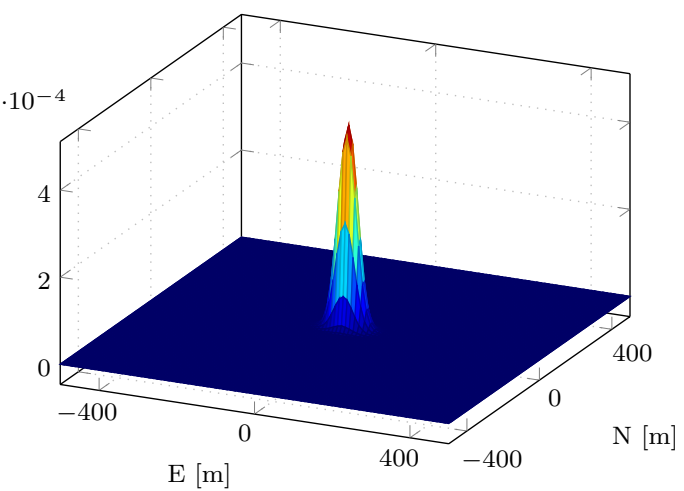

(d)

shaped region in Cartesian coordinates (dark blue, dark red). b The banana-shaped uncertainty regions are approximated by Gaussians. The fused target candidate is represented by its mean (red cross) and covariance (red ellipse). c Both Gaussians overlapping in ENU frame. d Merged Gaussian in ENU frame

at time $k$. In other words, the target measurement is described via the mean and its corresponding covariance. The natural coordinate frame for marine radar measurements is the polar domain, with the origin at the radar's global position. This implies that the measurement vector $\mathbf{z}_{k}^{s}$ equals $[r, \theta]^{\mathrm{T}}$, capturing the range $r$ and bearing $\theta$ of the target at time $k$ w.r.t. radar $s$. The uncertainty of the detection can be described in polar coordinates as

$\mathbf{R}_{k}^{s}=\left[\begin{array}{cc}\sigma_{s, r}^{2} & 0 \\ 0 & \sigma_{s, \theta}^{2}\end{array}\right]$,

with $\sigma_{s, r}$ and $\sigma_{s, \theta}$ being the expected standard deviation in range and bearing, respectively. For fusing two measurements from different radars they need to be represented in the same reference system, calling for an initial transformation from the local polar to the global Earth-Centered, 
Earth-Fixed (ECEF) frame. From this global frame, we are free to choose a reference point to transform the measurements back to a local Cartesian East-North-Up (ENU) plane. While the coordinate transformation of the measurement mean values is a straightforward procedure, special care must be taken with respect to the transformation of the measurement uncertainties. These are assumed to be Gaussian in the polar domain leading to banana-shaped uncertainty distributions in the local Cartesian plane as was visualized in Fig. 3. To avoid the non-trivial analytical expression of this projection, we approximate this probability distribution function (pdf) again with a Gaussian defined as

$\operatorname{pdf} \sim \mathcal{N}\left(\boldsymbol{\mu}=\left[\begin{array}{l}x_{k}^{s} \\ y_{k}^{s}\end{array}\right], \boldsymbol{\Sigma}=\tilde{\mathbf{C}}_{k}^{s}\right)$

with

$\tilde{\mathbf{C}}_{k}^{s}=\left[\begin{array}{cc}\sigma_{\tilde{x}}^{2} & 0 \\ 0 & \sigma_{\tilde{y}}^{2}\end{array}\right]$.

The standard deviations in $\tilde{x}$ and $\tilde{y}$ are computed from $\sigma_{\tilde{y}}=\sigma_{r}$ and $\sigma_{\tilde{x}}=r_{k} \sigma_{\theta}$. The former holds true, since radial and $\tilde{y}$-axis are equivalent. The latter approximates the uncertainty in the tangential direction $(\tilde{x}$-axis) with the arc length. Figure $3 \mathrm{~b}$ shows the outcome of this simplification by plotting the contour lines of the $3 \sigma$ threshold on top of the originally bended uncertainty region. The approximated Gaussians, however, are still aligned with the radial and tangential direction of their local radar preventing the fusion of both distributions. Thus, the misalignment needs to be compensated for each $\tilde{\mathbf{C}}_{k}^{s}$ by rotating the covariances back into the same frame. This transformation yields a generalized, non-diagonal covariance matrix

$\boldsymbol{C}_{k}^{s}=\left[\begin{array}{cc}\sigma_{x}^{2} & \rho \sigma_{y} \sigma_{x} \\ \rho \sigma_{x} \sigma_{y} & \sigma_{y}^{2}\end{array}\right]$,

aligned with the $x$ - and $y$-axes of the reference ENU frame. Any affine transformation $\boldsymbol{F}$ on the state vector $\boldsymbol{x}_{k}$ translates to

$\boldsymbol{C}_{k}^{s}=\boldsymbol{F} \tilde{\boldsymbol{C}}_{k}^{s} \boldsymbol{F}^{\mathrm{T}}$

in case of transforming the covariance to the same domain. Thus, to align the covariance matrix with the $x$ - and $y$-axis of the ENU reference frame we need to rotate the matrix $\tilde{\boldsymbol{C}}_{k}^{s}$ by the bearing angle $\theta^{s}$ according to

$\boldsymbol{C}_{k}^{s}=R\left(\theta^{s}\right) \tilde{\boldsymbol{C}}_{k}^{s} R\left(\theta^{s}\right)^{\mathrm{T}}$.

In this context, $R(\cdot)$ is defined as counter-clockwise rotation. With both target measurements being transformed and aligned to the same reference frame they can eventually be fused. In fact, very similar to the correction step of the Kalman Filter in [22] both Gaussians are collapsed into one. In general, the covariance and the shifted mean for fusing two Gaussian are obtained from

$\boldsymbol{C}_{k}=\boldsymbol{C}_{k}^{A}-\boldsymbol{C}_{k}^{A}\left[\boldsymbol{C}_{k}^{A}+\boldsymbol{C}_{k}^{B}\right]^{-1} \boldsymbol{C}_{k}^{A^{\mathrm{T}}}$

and

$\boldsymbol{\mu}_{k}=\boldsymbol{\mu}_{k}^{A}+\boldsymbol{C}_{k}^{A}\left[\boldsymbol{C}_{k}^{A}+\boldsymbol{C}_{k}^{B}\right]^{-1}\left[\boldsymbol{\mu}_{k}^{B}-\boldsymbol{\mu}_{k}^{A}\right]$.

The fusion process for two Gaussians is also shown in Fig. 3c, d using the identical scenario from before. To complete the process, the fused covariance $\boldsymbol{C}_{k}$ needs to be rotatedback into the original ENU reference frame, as it will be misaligned with its axes after applying Eq. 11 . The new orientation $\tilde{\theta}$ of $\boldsymbol{C}_{k}$ is obtained from eigenvalue decomposition. If one takes the eigenvector $\boldsymbol{v}=\left[v_{x}, v_{y}\right]^{\mathrm{T}}$ that corresponds to the largest eigenvalue of $\boldsymbol{C}_{k}, \tilde{\theta}$ is obtained with

$\tilde{\theta}=\arctan \left(\frac{v_{y}}{v_{x}}\right)$.

Note, that the angle $\tilde{\theta}$ is defined in counter-clockwise direction in this case, spanning from the $x$-axis to the eigenvector $v$ in the ENU frame. Hence, the negated $\tilde{\theta}$ has to be plugged in Eq. 10 for clockwise rotation of $\boldsymbol{C}_{k}$ to obtain the required estimate of the sensor noise covariance

$\tilde{\mathbf{R}}_{k}=R(-\tilde{\theta}) \boldsymbol{C}_{k} R(-\tilde{\theta})^{\mathrm{T}}$.

This concludes the description of the measurement-to-measurement association and fusion technique applied for two radars. Eventually, three different sets of target candidate measurements $\tilde{\mathcal{Z}}_{k}, \mathcal{Z}_{k}^{A \cup\{A \cap B\}}$ and $\mathcal{Z}_{k}^{B \cup\{A \cap B\}}$ are used to update the multi-target state estimate. The applied algorithm for multi-object tracking will be introduced in the follow-up section.

\subsection{Multi-target state estimation}

In general, the field of multi-target tracking (MTT) in the presence of multiple and in general imperfect sensors has been widely explored, ranging from classical enumerative to non-enumerative schemes. Algorithms representing the former category, such as GNN and JPDA filtering, integer programming or Multi Hypothesis Tracking (MHT), are well described in [2, 25, 34] and [24]. More recent work has also applied random finite set (RFS) theory to MTT yielding the Probability Hypothesis Density (PHD) or Cardinalized PHD (CPHD) filters [28]. The extension of these frameworks to the case of distributed target tracking has 
for instance led to the generic formulation of Distributed MHT [11].

\subsubsection{Target dynamics and measurement model}

Typically, the state vector $\mathbf{x}_{k-1}^{t}$ of the $t$ th target to the next time frame $k$ is propagated through a non-linear motion model following

$\mathbf{x}_{k \mid k-1}^{t}=f^{i}\left(\mathbf{x}_{k-1}^{t}, \epsilon_{k}^{q}\right)$,

with $\boldsymbol{\epsilon}_{k}^{q} \sim \mathcal{N}\left(\mathbf{0}, \Sigma=\mathbf{Q}_{k}^{i}\right)$ and no further control input given. To distinguish between different dynamic models in the upcoming section superscript $i$ is introduced to the nonlinear function $f^{i}(\cdot)$. The predicted state estimate $\mathbf{x}_{k \mid k-1}^{t}$ is to be corrected by evaluating the residual between the actual radar measurement $\mathbf{z}_{k}$ associated to the $t$ th target and the predicted measurement following the general formulation

$\hat{\mathbf{z}}_{k \mid k-1}^{s}=h^{s}\left(\mathbf{x}_{k \mid k-1}^{t}, \boldsymbol{\epsilon}_{k}^{r}\right)$

with $\boldsymbol{\epsilon}_{k}^{r} \sim \mathcal{N}\left(\mathbf{0}, \Sigma=\mathbf{R}_{k}^{s}\right)$. In case of updating $\mathbf{x}_{k \mid k-1}^{i}$ with individual radar measurements in polar domain $h^{s}(\cdot)$ is defined as

$$
\begin{aligned}
& h^{s}\left(\mathbf{x}_{k \mid k-1}^{i}, \boldsymbol{\epsilon}_{k}^{r}\right) \\
& \quad=\left[\begin{array}{c}
\sqrt{\left(p_{e, k \mid k-1}-p_{e}\right)^{2}+\left(p_{n, k \mid k-1}-p_{n}\right)^{2}} \\
\arctan \left(\frac{p_{e, k \mid k-1}-p_{e}}{p_{n, k \mid k-1}-p_{n}}\right)
\end{array}\right]+\epsilon_{k}^{r},
\end{aligned}
$$

with $\left\{p_{e}, p_{n}\right\}$ the 2D reference coordinates of the radar system in the ENU frame of the tracked vessel. The sensor uncertainty $\sigma_{r}$ and $\sigma_{b}$ in range and bearing, respectively, is modeled with

$\mathbf{R}_{k}^{s}=\left[\begin{array}{cc}\sigma_{r}^{2} & 0 \\ 0 & \sigma_{b}^{2}\end{array}\right]$.

\subsubsection{The IMM-JPDA filter}

In consideration of the inherent trade-off between complexity and tracking performance the JPDA framework was chosen, being combined with an IMM filter to capture different target dynamics. First introduced in [14], the key feature of the JPDA is the computation of conditional probabilities of joint association events:

$A(k)=\bigcap_{j=1}^{M} A_{j t}(k)$,

with respect to the current time $k$, in which $A_{j t}(k)$ represents the event of the $j$ th measurement originating from target $t$, with $1 \leq j \leq M$ and $0 \leq t \leq N$. $M$ refers to the number of measurements at time $k, N$ to the number of known targets and $t_{j}$ is the target index the $j$ th measurement is associated to. With $t=0$ the specific case of a measurement originating from clutter is also being considered. In contrast to a nearest neighbor (NN) association rule, the JPDA also accounts for situations in which a single measurement has a certain likelihood to originate from multiple targets at the same time (details can be found in [14] and [2]). It can be seen quite easily that the considerations of all joint events results in a combinatoric growth in complexity for more measurements and more targets. To reduce the initial set of feasible association events a validation matrix $\mathbf{V}$ is usually computed first, which is a binary matrix of dimensionality $M \times(N+1)$ representing all possible associations between measurements and targets. Whether the single matrix element $v_{j t}$ is set to either 0 or 1 depends on validation gating, i.e. the condition

$\left[\mathbf{z}_{j, k}-\hat{\mathbf{z}}_{t_{j}, k \mid k-1}\right]^{\mathrm{T}} \mathbf{S}_{t_{j}, k}^{-1}\left[\mathbf{z}_{j, k}-\hat{\mathbf{z}}_{t_{j}, k \mid k-1}\right]<\gamma$.

needs to be fulfilled for $v_{j t}$ to become 1 . Volume $V$ of this gate depends on the inverse of the innovation covariance $\mathbf{S}_{t_{j}, k}^{-1}$. A comprehensive comparison of different variations on the standard JPDA filtering can be found in [34]. In this study, an extension known as IMM-JPDA is applied that was initially proposed by [6] and extended to the multi-sensor case in [40]. The IMM filter was introduced in [5] to adopt to quickly changing target dynamics, which is also relevant in the case of tracking arbitrary object in the vicinity of a marine radar. In fact, a finite set of kinematic models is run in parallel in the IMM. In contrast to hard switching schemes, the output of the IMM combines the weighted target state estimates, where the weights are based on the likelihood of each model to explain the current measurement data. The application of IMM filtering to maritime target tracking has already been shown to be beneficial in [36]. In our case, we expect three dominating dynamic modes to be tracked, namely static-, straight-path- and turn-maneuver-based motion. Thus, a set of three dynamic models was defined, referred to as Constant Position (CP), Constant Velocity (CV) and Constant Turn Rate Velocity (CTRV) model. The respective target state vectors are defined as

$\mathbf{x}_{k}^{\mathrm{CP}}=\left[p_{e, k}, p_{n, k}\left[^{\mathrm{T}}\right.\right.$,

$\mathbf{x}_{k}^{\mathrm{CV}}=\left[p_{e, k}, p_{n, k}, \psi_{k}, v_{k}\right]^{\mathrm{T}}$,

$\mathbf{x}_{k}^{\mathrm{CTRV}}=\left[p_{e, k}, p_{n, k}, \psi_{k}, v_{k}, \dot{\psi}_{k}\right]^{\mathrm{T}}$,

with $\left\{p_{e, k}, p_{n, k}\right\}$ being the $2 \mathrm{D}$ position coordinates in the local ENU frame, $\psi_{k}$ the course over ground (COG), $v_{k}$ the speed over ground (SOG) and $\dot{\psi}_{k}$ the turn rate at time $k$. 
For detailed definitions of the non-linear process models $f^{i}(\cdot)$ for CV and CTRV see [38]. Careful attention needs to be paid to the augmentation of state vectors of different dimensions, when it comes to model mixing within the IMM. In this study, we follow a strategy described in [15] for unbiased mixing of different process models with varying state dimension. Deviating from the common formulation of either IMM or JPDA, which both use Extended Kalman Filtering (EKF) to adopt to non-linearities in the dynamic and sensor models, the Unscented Kalman Filter (UKF) is deployed instead (see [21]). It turns out that in case of radar measurement updates the UKF is more robust against situations, in which the assumptions of a mildly non-linear problem and Gaussian sensor noise are violated [7]. The implementation in this work is based on the recursive stepby-step algorithm from [40], incorporating measurements from different sensors sequentially during the update stage of the IMM-JPDA filter.

\subsubsection{Track management}

In general, the JPDA filter is subject to several assumptions. Most important for our application is that the finite set of targets to be tracked is assumed to be known. This means that neither track initialization nor track pruning are covered by the standard formulation of JPDA. To overcome these restrictions it is suggested to apply an $M$-of- $N$ rule in [1], which was implemented similar to [7] from the following steps:

\section{Track initialization:}

- For each radar scan, every unassigned target candidate measurement becomes a tentative track. The gate assigned to this track accounts for the (assumed) maximum velocity and sensor uncertainty. This is a rather conservative bound.

- If a target candidate from the next radar scan falls within the gate of a tentative track, it becomes a preliminary track. In case a tentative track is not supported by any detection in the next time frame it is removed.

- For each preliminary track a UKF is initialized propagating the target state through a $\mathrm{CV}$ dynamic model.

- If a preliminary track is confirmed for $M$ out of the next $N$ radar scans, it becomes a confirmed track. If this is not the case it is dropped.

- Each confirmed track will be tracked in the IMMJPDA filter.

2. Track termination:
- In case a confirmed track is not updated for $M_{t}$ out of $N_{t}$ consecutive radar scans it is terminated. Index $t$ denotes the difference between parameters $M$ and $N$ from the initialization process.

- A confirmed track will also be terminated, in case the corresponding error state covariance exceeds predefined thresholds in position and/or velocity.

\subsubsection{MTT performance assessment with OSPA metric}

In [35] the Optimal Subpattern Assignment (OSPA) metric was introduced. It is considered as the state-of-the-art method for MTT performance assessment. The OSPA metric yields several characteristics that make it attractive for MTT performance assessment:

- It has a physical interpretation.

- It captures multi-target state and cardinality errors meaningfully.

- The OSPA metric depends on only two tuning parameters: the order $p$ and cutoff parameter $c$.

- It has a little computational demand.

Consider two finite subsets $X=\left\{x_{1}, \ldots, x_{m}\right\}$ and $Y=\left\{y_{1}, \ldots, y_{n}\right\}$ within $W$, where $m, n \in \mathbb{N}_{0}=\{0,1,2, \ldots\}$, and denote the set of permutations on $\{1,2, \ldots, k\}$ for any $k \in \mathbb{N}=\{1,2, \ldots\}$ by $\Pi_{k}$. The OSPA metric is then defined as

$\bar{d}_{p}^{(c)}(X, Y)$

$:=\left(\frac{1}{n}\left(\min _{\pi \in \Pi_{n}} \sum_{i=1}^{m} d^{(c)}\left(x_{i}, y_{\pi(i)}\right)^{p}+c^{p}(n-m)\right)\right)^{(1 / p)}$,

with $d^{(c)}\left(x_{i}, y_{\pi(i)}\right)=\min (c, d(x, y))$ denoting the distance between $x$ and $y$ cutoff at $c>0$. This definition holds for $m \leq n$. In case of $m>n$ one substitutes $\bar{d}_{p}^{(c)}(X, Y)$ by $\bar{d}_{p}^{(c)}(Y, X)$. According to [35], the impact of localization and cardinality errors to the overall metric can be expressed as

$\bar{e}_{p, \mathrm{loc}}^{(c)}(X, Y):=\left(\frac{1}{n} \min _{\pi \in \Pi_{n}} \sum_{i=1}^{m} d^{(c)}\left(x_{i}, y_{p i(i)}\right)^{p}\right)^{1 / p}$

and

$\bar{e}_{p, \text { card }}^{(c)}(X, Y):=\left(\frac{c^{p}(n-m)}{n}\right)^{(1 / p)}$. 
Table 1 Summary of dynamic and sensor model noise settings

\begin{tabular}{ll} 
(a) Dynamic models & \\
CP & $\sigma_{p_{e}}=0.1 \mathrm{~m} / \mathrm{s}$, \\
& $\sigma_{p_{n}}=0.1 \mathrm{~m} / \mathrm{s}$ \\
CV & $\sigma_{v}=0.01 \mathrm{~m} / \mathrm{s}^{2}$, \\
& $\sigma_{\psi}=0.01^{\circ} / \mathrm{s}$ \\
CTRV & $\sigma_{v}=0.1 \mathrm{~m} / \mathrm{s}^{2}$, \\
& $\sigma_{\dot{\psi}}=0.1^{\circ} / \mathrm{s}^{2}$ \\
(b) Sensor models & \\
Radar $A$ & $\sigma_{r}=25 \mathrm{~m}$, \\
& $\sigma_{b}=1^{\circ}$ \\
Radar $B$ & $\sigma_{r}=30 \mathrm{~m}$, \\
& $\sigma_{b}=0.75^{\circ}$ \\
\hline
\end{tabular}

Table 2 Summary of IMM-JPDA settings

\begin{tabular}{ll}
\hline JPDA type & Parametric \\
\hline (a) General JPDA settings & \\
Spatial density $\lambda$ & $10^{-9}$ \\
$P_{d \text {,radar } A}$ & 0.9 \\
$P_{d \text {,radar } B}$ & 0.8 \\
$P_{d, \text { fused }}$ & 0.98 \\
(b) IMM settings [5] & \\
$\mathbf{T}=\left[\begin{array}{ccc}0.9 & 0.05 & 0.05 \\
0.05 & 0.9 & 0.05 \\
0.05 & 0.05 & 0.9\end{array}\right]$ &
\end{tabular}

Table 3 Settings for parameters similar in both image-based target detectors

\begin{tabular}{lll}
\hline & Radar $A$ & Radar $B$ \\
\hline Min. threshold & 120 & 150 \\
Min. blob area & $21 \mathrm{px}$ & $51 \mathrm{px}$ \\
\hline
\end{tabular}

\section{Results}

In the previous sections, a framework for multi-target acquisition and tracking based on multi-radar fusion was presented. Real-world data will be used in the following for general proof-of-principle and performance evaluation of two different radar image-based target detectors. At first, the measurement campaign from which the data were obtained will be outlined. Second, the overall performance in multi-target state estimation for different configurations of the framework will be analyzed and discussed. The summary of relevant settings for the IMM-JPDA filter and image-based detectors w.r.t. this analysis is listed in Tables 1, 2 and 3 .

\subsection{Multi-target, multi-radar experiment}

In June 2016, a dedicated measurement campaign was conducted in the Baltic Sea close to the island of Hiddensee, Germany (Fig. 4). For evaluation purposes, a 30-min long snippet from the entire 3-day campaign was selected. The scenario within this subset can be characterized as follows:

- Three vessels actively participated in the measurement campaign:

- two chartered ships: BALTIC TAUCHER II (diving support vessel) and WIND PROTECTOR (tender vessel),

- one external ship: THEO FISCHER (salvage vessel, which was still on duty),

- all of these ships were equipped with AIS transmitters.

- Two unidentified (not visible in AIS) fishing boats were passing by.

- Four buoys were anchored in predefined positions to mark the campaign area; all of them were equipped with AIS transmitters.

- Two radars were monitoring the test area installed on the anchored WIND PROTECTOR and the mobile BALTIC TAUCHER II; Fig. 5a, b shows the identical scene captured by both radars from different aspect angles,

- The THEO FISCHER performed dynamic maneuvers within the experiment area.

From the description above, it can be concluded that four mobile and five static objects are needed to be detected and tracked. The latter set comprised the buoys and the WIND PROTECTOR, the former both fishery vessels, the THEO FISCHER and the BALTIC TAUCHER II. This implies, that each of the vessels acting as radar platforms was part of the multi-target scenario itself, as either one was seen by the other. During this multi-target, multi-radar scenario we applied the proposed algorithm from Fig. 1 testing the performance of two different radar image processing techniques: first, the extracted measurements from both radars were associated and fused, if the association pairs passed the Bhattacharyya test. Second, a centralized IMM-JPDA tracker is updated on either if available the fused measurements or individual radar measurements. For performance characterization in terms of the quantitative OSPA metric the multi-target state reference is required. In the absence of precise position information for all of the objects in sight we used the AIS trajectories available as reference. In fact, except for the two fishing vessels all other objects transmitted AIS messages. This means, the OSPA metric shown in later figures was computed from a reduced multi-target 


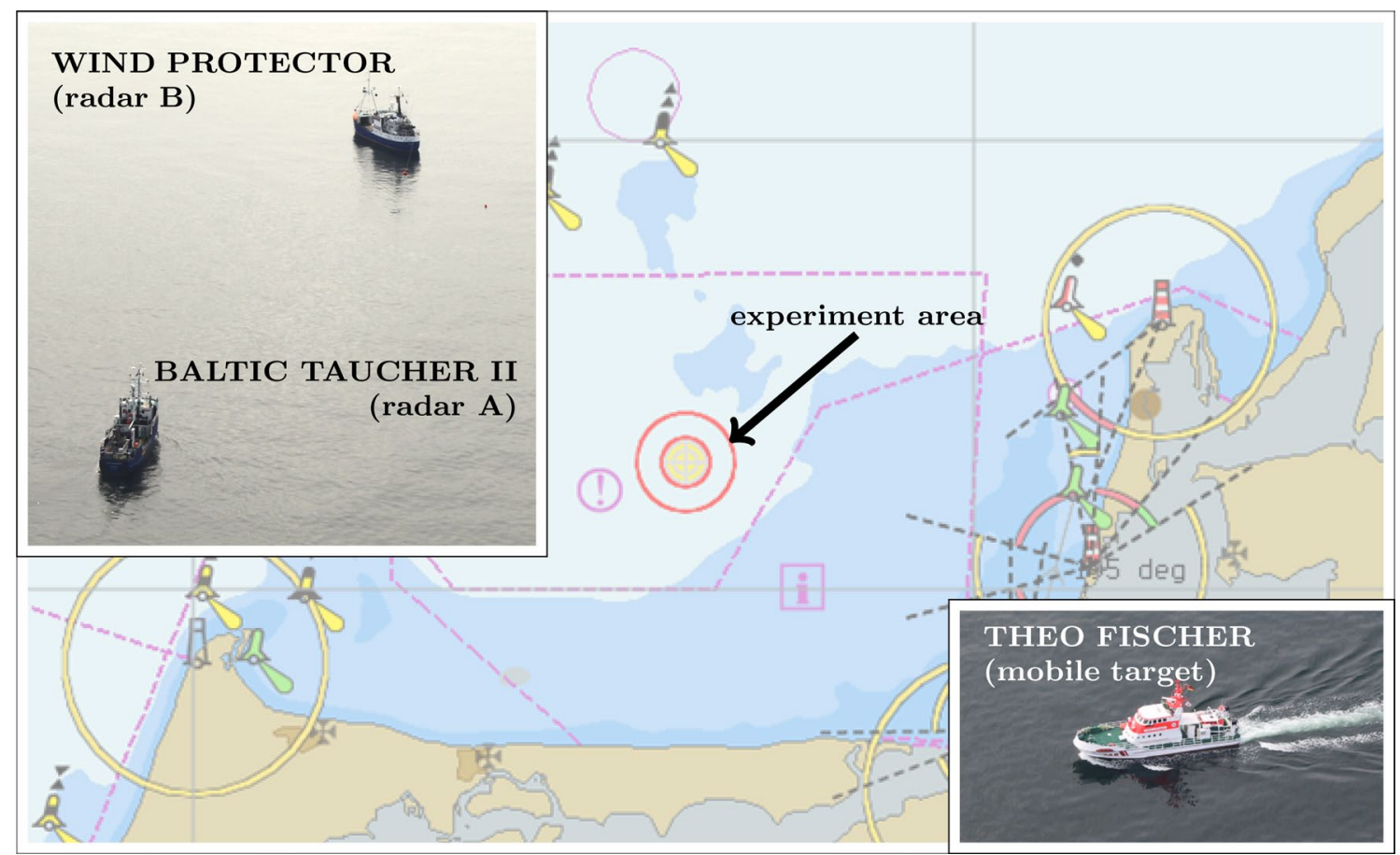

Fig. 4 The measurement campaign was conducted in the Baltic Sea close to Hiddensee, Germany. Three vessels actively participated, the BALTIC TAUCHER II as well as the WIND PROTECTOR, which acted as sensor platforms carrying a radar each. The search-and-rescue vessel THEO FISCHER as a mobile target, which was making sharp turns mixed with straight path motion

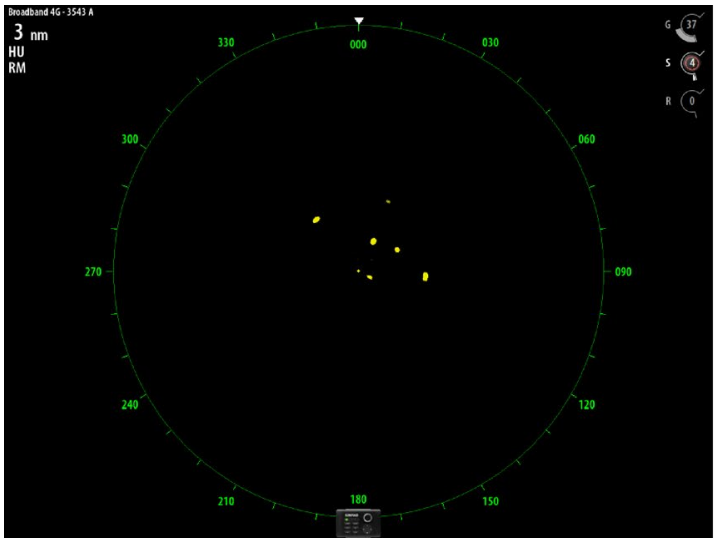

(a)

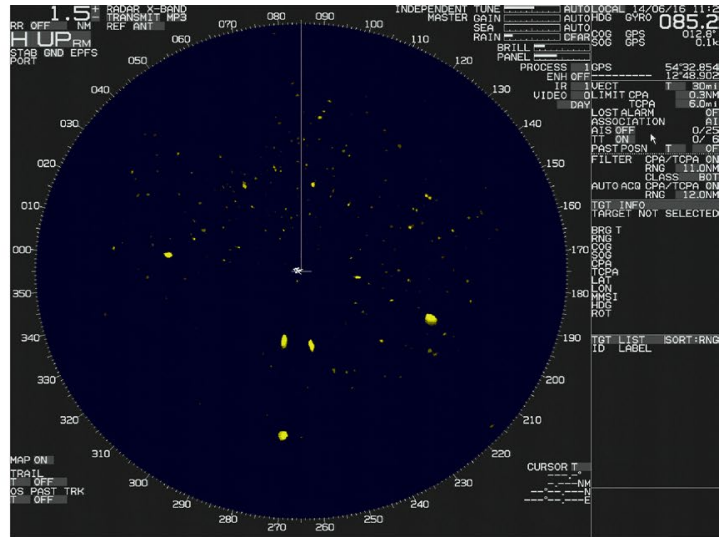

(b)

Fig. 5 Responses from radar $A$ and $B$ at arbitrary time instance $k$ for the identical situation during measurement campaign. a Response from radar $A$ aboard BALTIC TAUCHER II. b Same situation observed by radar $B$ aboard WIND PROTECTOR

reference set (two mobile/five static objects), while the plots in Figs. 6 and 7 show the complete multi-target state estimate in the 2D position domain. Note, that the AIS reference data are limited in its accuracy up to several meters as well.

\subsection{Multi-target state estimate for BLOB detector}

In Fig. 6 the resulting tracks are shown when applying the BLOB detector to extract target measurements from both radar responses. The local Cartesian plane is centered at the position of the anchored WIND PROTECTOR. It can be seen that the framework acquires and tracks all nine targets, i.e., four dynamic (target-IDs $\{1,4,6,9\}$ ) and five static objects (four buoys with target-IDs $\{2,3,7,8\}$; one anchored vessel with ID 5). Note again, that not all objects can be seen by both radars but are still included in the tracking process. For instance, track \#4 describes the trajectory of the BALTIC TAUCHER II acting as radar platform itself, whereas 


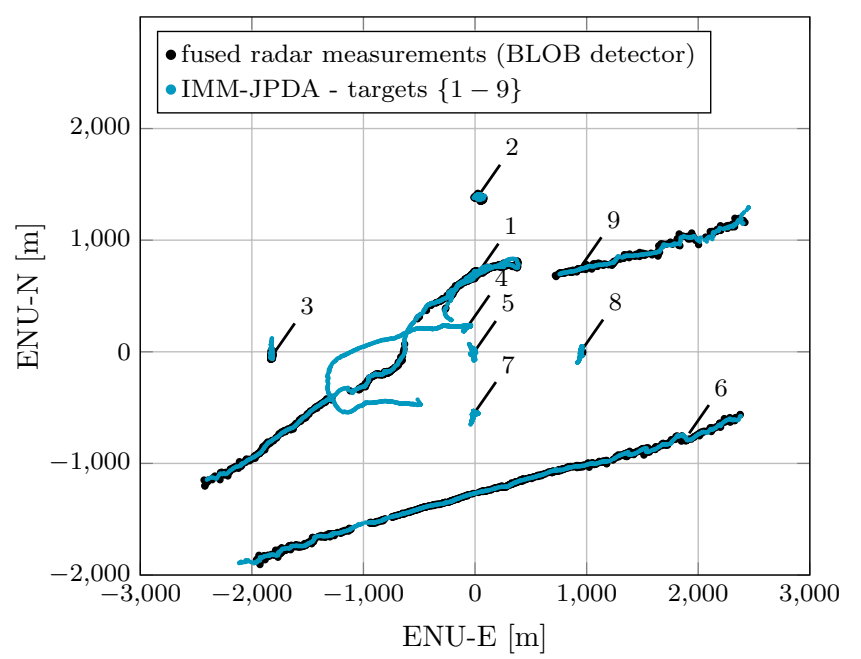

(a)

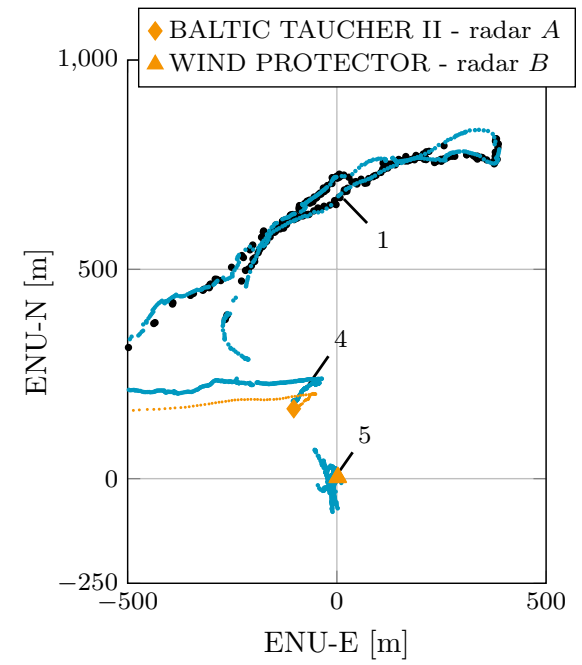

(b)
Fig. 6 Resulting multi-target state estimate from IMM-JPDA filtered radar target measurements. The target candidates were extracted by applying the BLOB detector to the plain radar images. The tracks (light blue lines) are plotted in a 2D local plane centered at the position of WIND PROTECTOR. The fused radar measurements are represented by black dots underneath the estimated tracks. In this scenario four mobile and five stationary (four buoys, one anchored vessel) targets were visible in radar. a Full scenario on 2D position plane with five static and four mobile targets. b Zoom to visualize the radar platform positions

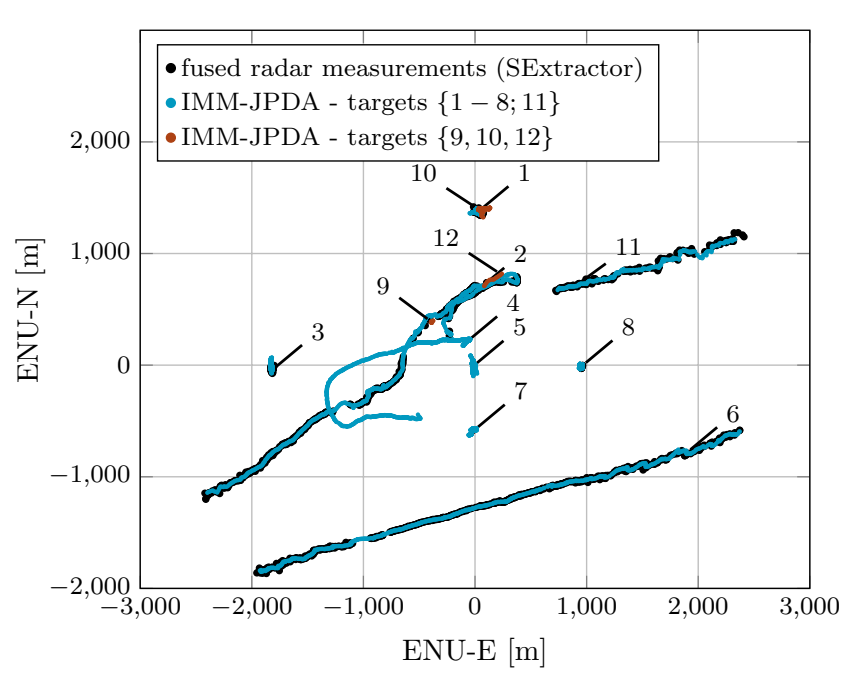

(a)

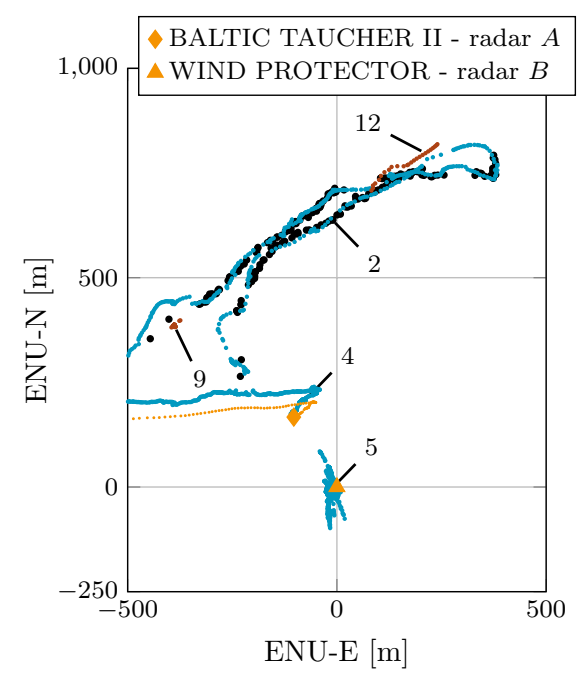

(b)
Fig. 7 Resulting tracks for the same scenario in case of applying the SExtractor to the plain radar images for target candidate extraction. The lines in light blue denote again the tracks that are correctly tracked, whereas the red lines refer to the tracks that are established in

track \#5 refers to the WIND PROTECTOR also carrying a radar. Both are only visible in the radar response of the other vessel. Figure $6 \mathrm{a}$ shows the full scale scenario in the $2 \mathrm{D}$ plane of the ENU frame, covering an area of roughly $3 \times 4$ square miles. The IMM-JPDA filtered tracks are plotted as blueish dots, while the black dots represent the mean of the fused radar measurements. For that reason, the tracks with parallel to existing one. a Full scenario on 2D position plane with five static and four mobile targets. b Zoom to visualize the radar platform positions

IDs $\{4,5,7\}$ are plotted standalone, as no fused measurements were available. Figure $6 \mathrm{~b}$ shows a zoomed in picture of the area around the anchored vessel WIND PROTECTOR. It can clearly be seen that fused radar measurements are not available throughout the entire time for track \#1, while the vessel is still being tracked based on the individual radar measurements. We can also see a systematic offset 


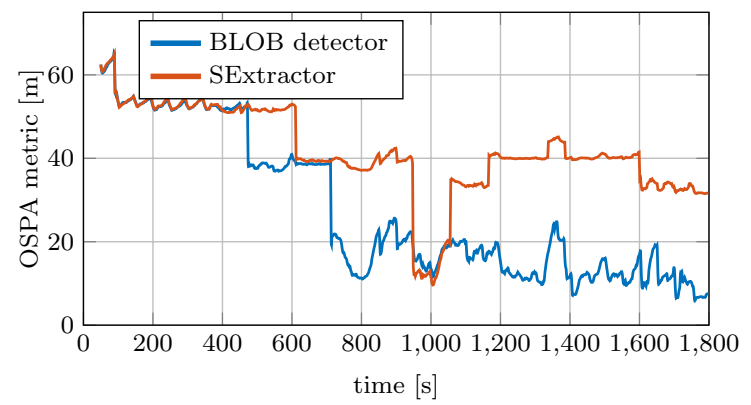

(a)

Fig. 8 Comparison of multi-target state estimation for two different target candidate extractors. The OSPA metric is plotted against time, with the results of the BLOB detector-based estimation plotted in

between track \#4 and its actual trajectory (orange), which shows some drawbacks of radar image-based tracking. First of all, the radar itself might be biased due to the shape of the reflective area of the object. Second, working with radar images may introduce additional error sources such as signal distortions during transformation to the image domain. Theoretically, the impact of these errors could be minimized by calibrating such a system first, similar to optical sensors.

\subsection{Multi-target state estimate for SExtractor detector}

Figure 7 shows the resulting tracks of the IMM-JPDA filter for the SExtractor. It can be seen that the IMM-JPDA based on the SExtractor detections results in 12 target tracks including again all four dynamic objects (target-IDs: $\{[2,12,9], 4,6,9,11\})$ as well as all five static targets (four buoys with IDs: $\{[1,10], 3,7,8\}$; one anchored vessel with ID: 5). The main difference in Fig. 6 is the fact that some of the objects are tracked in parallel by different tracks (indicated by the square brackets in the previous mentioning). The tracks with IDs 1 and 10 track the same static buoy, and the tracks with IDs 2, 12 and 9 track the same mobile vessel. This is most likely due to an increased sensitivity of the SExtractor towards clutter measurements yielding more false detections. If these fall repeatedly in the initial validation gates of tentative or preliminary tracks, respectively, they are picked up as confirmed tracks and depleted in case they are not supported by measurements anymore (track IDs 9 and 12).

\subsection{Performance comparison with OSPA metric}

For analysis reasons, two different configurations of the OSPA metric were chosen that only differ in the cut-off parameter. While the order $p$ was set constantly to 2 , the penalty for a cardinality mis-match altered between $c=250 \mathrm{~m}$

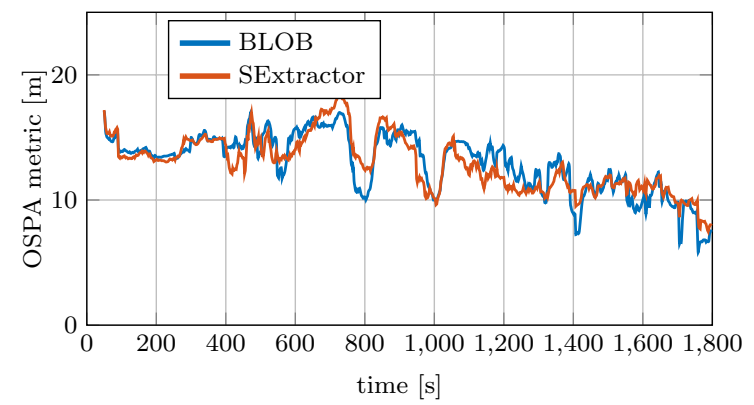

(b)

blue and the SExtractor plotted in red. a OSPA metric for $c=250 \mathrm{~m}$. b OSPA metric for $c=50 \mathrm{~m}$

and $c=50 \mathrm{~m}$, giving a high or low weight to these kinds of errors. Both configurations are shown here, as we do not wish to anticipate conclusions in one way or the other. However, in maritime situation awareness we would typically assume the correct assessment of the number of objects in sight to be prioritized above accuracy. The results of the OSPA metric computed from Eq. 24 are shown in Fig. 8. The plot illustrates the overall error in multi-target estimation for both filter configurations and allows a quantitative comparison. The subplots of Fig. 9 give a detailed view on the impact of cardinality and localization error to the overall score. In terms of the different OSPA configurations Figs. 8a, 9a, c show the result for the cut-off parameter $c$ set to $250 \mathrm{~m}$. Figures $8 \mathrm{~b}, 9 \mathrm{~b}, \mathrm{~d}$ depict the same scores for $c=50 \mathrm{~m}$. In all of these graphs, it can be seen that the framework conditioned on target measurements extracted from the BLOB detector performs clearly better in terms of estimated cardinality (tracking the correct number of targets) while the SExtractor has a slight advantage in the accuracy, i.e., localization domain. In fact, the shape of the cardinality error stays constant, only the scale, i.e., the impact, to the overall metric changes. The localization error, however, also changes in shape and absolute numbers with respect to an alternating $c$. Not only the sawtooth-like curve from Fig. 9c between 100 and $400 \mathrm{~s}$ disappears in Fig. 9d, also the pronounced peak from the BLOB detector driven localization error at $1300 \mathrm{~s}$ vanishes. This behavior is explained from Eq. 25, which is dependent on the minimized cost over the distance matrix between estimate and reference being cutoff at $c>0$. This means, state errors larger than $c$ do not account to the overall localization error. It has to be noted that both image processing methods can probably be tuned to yield better performance scores for this specific data set. Especially the SExtractor offers a vast range of parameters that were not fully exploited. The optimization of these settings, however, was out of the scope of this paper. If the more complex processing algorithm of the SExtractor is 


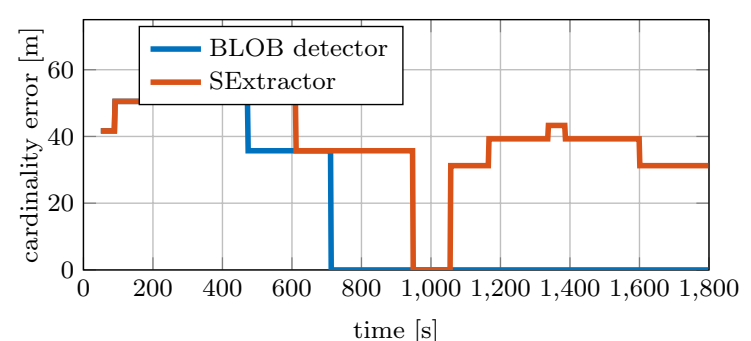

(a)

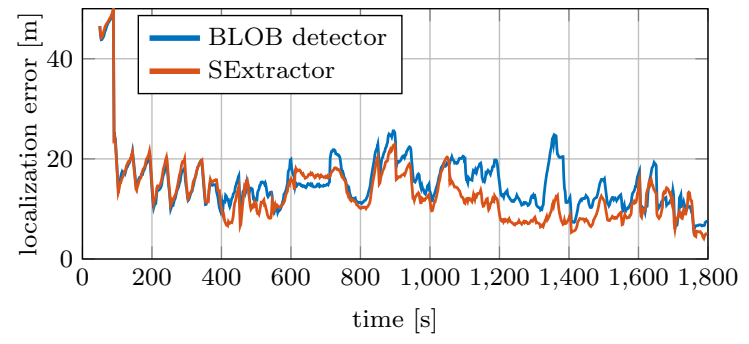

(c)

Fig. 9 The cardinality and localization errors $\bar{e}_{p, \text { card }}^{(c)}(X, Y)$ and $\bar{e}_{p, \text { loc }}^{(c)}(X, Y)$ for both multi-target state estimates for either $c=250 \mathrm{~m}$ and $c=50 \mathrm{~m}$, respectively. The results of the BLOB detector-based

actually superior to the implementation of BLOB detector in OpenCV for the majority of cases, this has to be investigated in future studies. This work was devoted to demonstrate not only the working principle of the radar image-based processing in the maritime domain but also the benefits from the modular design of the overall framework for object acquisition and tracking.

\subsubsection{Impact of sensor network configurations}

Besides radar image-based processing, another key element of the proposed framework is the radar measurement fusion prior to the actual target acquisition and tracking. In Fig. 10, we show that the outcome of this approach is actually worth the effort. In comparison with the proposed type III configuration, we show the result for a sensor network in which all the individual radar measurements are used to update the IMM-JPDA tracker directly. This time the OSPA metric was set up with $p=2$ and $c=250 \mathrm{~m}$ only to give more emphasis on cardinality errors. In fact, the proposed type III configuration yields a better performance in cardinality and localization errors. This is most likely due to the increased number of target candidates in case measurements originating from the identical targets are not fused a priori. In this case, the overall framework with the chosen settings is more sensitive in terms of target acquisition yielding several tracks following the same object simultaneously. This phenomenon is also responsible for the increase in the localization error between $\sim 140$ and $420 \mathrm{~s}$. The $\bar{e}_{p, \text { loc }}^{(c)}(X, Y)$ reflects that not all

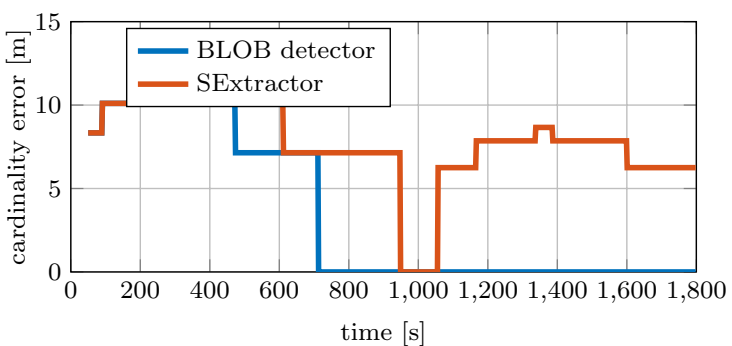

(b)

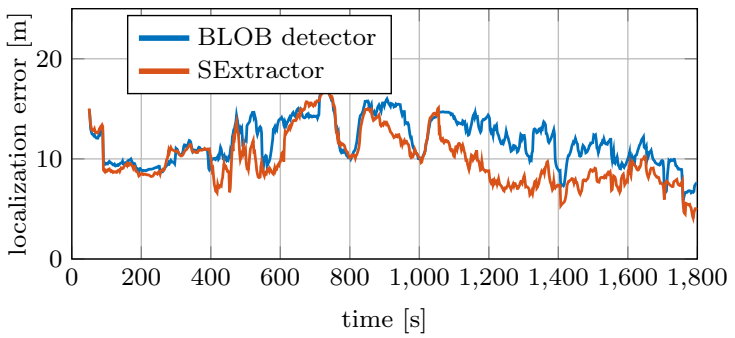

(d)

estimation are plotted in blue and the SExtractor in red. a Cardinality errors for $c=250 \mathrm{~m}$. b Cardinality errors $c=50 \mathrm{~m}$. c Localization errors for $c=250 \mathrm{~m}$. d Localization errors $c=50 \mathrm{~m}$

objects are seen in radar at that time but that multiple tracks were initialized for identical objects instead. These additional tracks are assigned to the remaining reference targets leading to the increase in localization error.

\section{Conclusion and outlook}

In this work, we have presented a generic framework for maritime situation assessment based on multiple marine radars. In contrast to classical radar signal processing, we apply techniques from computer vision to extract target candidates from the radar image itself. These target measurements are associated and fused if they pass a similarity test. Both sets of measurements, fused and remaining individual radar measurements, are used to update a centralized multitarget tracker. All target candidates that cannot be associated to existing tracks are forwarded to the track management module, yielding tentative or preliminary tracks. In this work, we compared the performance in multi-target state estimation for two different radar image processing techniques. It was shown that the OpenCV implementation of a BLOB detector slightly outperforms the SExtractor; a method very popular for star detection in astronomy. It has to be noted that especially the SExtractor offers many more tuning parameters that were not fully exploited in this study. Our main intention was clearly to prove the working principle of the overall framework by substituting one imagebased method for the other. Additionally, the benefit of radar 


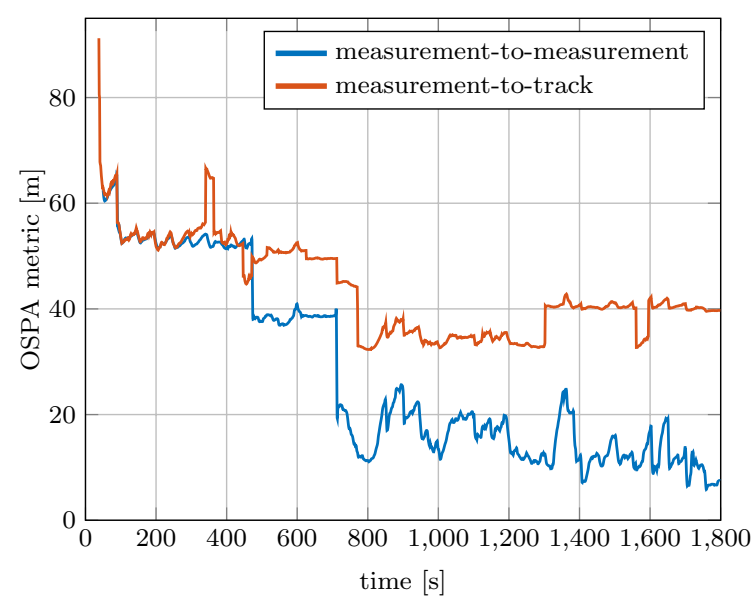

(a)

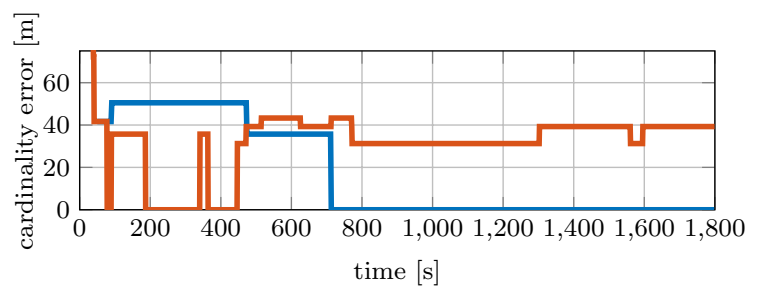

(b)

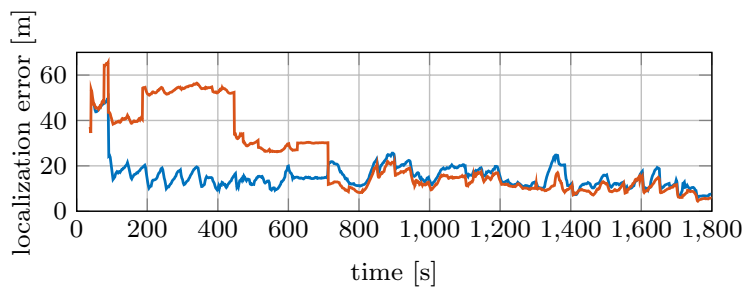

(c)
Fig. 10 Performance comparison for different fusion strategies for multiple radars. The blue curve represents the proposed type III configuration according to the flow chart in Fig. 1. The red curve origins from a measurement-to-track association rule, in which all radar measurements are used to update the centralized tracker directly.

measurement-to-measurement association and fusion prior to the actual tracking was demonstrated. The base scenario used for evaluation was taken from a real-world measurement campaign, comprising four mobile and five static objects being monitored by two distributed radars.

In future, we will direct our research towards a more generic scheme for cooperative situation assessment, to exploit the diversity of information within a maritime network of distributed vessels acting as sensor platforms.

Acknowledgements We thank the crew of the BALTIC TAUCHER II for their support. We also thank our colleagues Uwe Netzband, Stefan Gewies and Carsten Becker for their helpful assistance during the measurement campaign.

\section{References}

1. Bar-Shalom, Y., Li, X.R.: Multitarget-Multisensor Tracking: Principles and Techniques. Yaakov Bar-Shalom, Romania (1995)

2. Bar-Shalom, Y., Daum, F., Huang, J.: The probabilistic data association filter. IEEE Control Syst. Mag. 29, 82-100 (2009)

3. Bertin, E., Arnouts, S.: SExtractor: software for source extraction. Astron. Astrophys. Suppl. 117, 393-404 (1996). https://doi. org/10.1051/aas:1996164

4. Bhattacharyya, A.: On a measure of divergence between two multinomial populations. Sankhy 7(4), 401-406 (1946)

5. Blom, H.A.P., Bar-Shalom, Y.: The interacting multiple model algorithm for systems with Markovian switching coefficients. IEEE Trans. Autom. Control. 33, 780-783 (1988)

6. Blom, H.A.P., Bloem, E.A.: Combining IMM and JPDA for tracking multiple maneuvering targets in clutter. In: Proceedings of the Fifth International Conference on Information Fusion,
From the OSPA metric we can deduce that the type III configuration outperforms the measurement-to-track association regime for this setup. a OSPA metric for $c=250 \mathrm{~m}$ and $p=2$. b OSPA cardinality errors for $c=250 \mathrm{~m}$ and $p=2$. $\mathrm{c}$ OSPA localization errors for $c=250 \mathrm{~m}$ and $p=2$

2002, vol. 1, pp. 705-712 (2002). https://doi.org/10.1109/ ICIF.2002.1021224

7. Braca, P., Vespe, M., Maresca, S., Horstmann, J.: A novel approach to high frequency radar ship tracking exploiting aspect diversity. In: 2012 IEEE International Geoscience and Remote Sensing Symposium (IGARSS), pp. 6895-6898 (2012)

8. Briggs, J.: Target Detection by Marine. Radar Electromagnetics and Radar Series. Institution of Engineering and Technology, London (2004)

9. Burmeister, H.C.: Autonomous Navigation Results from the Munin Testbed. Autonomous Ship Technology Symposium, Amsterdam (2016)

10. Byun, S.H., Lee, H.J., Yoon, H.K., Gong, D.S., Lee, C.M., Kang, C.G.: Implementation of multi-vessel tracking algorithm using multiple marine radar systems. In: OCEANS '04. MTTS/ IEEE Techno-ocean '04, vol. 4, pp. 2242-2248 (2004). https:// doi.org/10.1109/OCEANS.2004.1406498

11. Coraluppi, S., Carthel, C., Wu, C., Stevens, M., Douglas, J., Titi, G., Luettgen, M.: Distributed MHT with active and passive sensors. In: 2015 18th International Conference on Information Fusion (Fusion), pp. 2065-2072 (2015)

12. Da Costa, G.S.: Basic photometry techniques. In: Howell, S.B. (ed.) Astronomical CCD Observing and Reduction Techniques, Astronomical Society of the Pacific Conference Series, vol. 23. ASP, San Francisco p. 90 (1992)

13. Drummond, O.E., Blackman, S.S.: Challenges of developing algorithms for multiple sensor, multiple target tracking. Proc. SPIE Signal Data Process. Small Targets 1096, 244-257 (1989). https://doi.org/10.1117/12.960358

14. Fortmann, T., Bar-Shalom, Y., Scheffe, M.: Sonar tracking of multiple targets using joint probabilistic data association. IEEE J. Ocean. Eng. 8(3), 173-184 (1983). https://doi.org/10.1109/ JOE.1983.1145560

15. Glass, J.D., Blair, W.D., Bar-Shalom, Y.: IMM estimators with unbiased mixing for tracking targets performing coordinated turns. In: Proceedings IEEE Aerospace Conference (2013) 
16. Guerriero, M., Willett, P., Coraluppi, S., Carthel, C.: Radar/AIS data fusion and SAR tasking for maritime surveillance. In: International Conference on Information Fusion, vol. 11 (2008)

17. Han, KTM, Uyyanonvara, B.: A survey of blob detection algorithms for biomedical images. In: 2016 7th International Conference of Information and Communication Technology for Embedded Systems (IC-ICTES), pp. 57-60 (2016). https://doi. org/10.1109/ICTEmSys.2016.7467122

18. Heymann, F., Banyś, P., Noack, T.: A pilot study of the advantage of radar image data over ARPA based position and bearing. In: European Navigation Conference 2014 (2014)

19. International Maritime Organization (IMO) (1974) International Convention for the Safety of Life at Sea (SOLAS)

20. Isard, M., MacCormick, J.: BraMBLe: a Bayesian multiple-blob tracker. Eighth IEEE Int. Conf. Comput. Vis. 2, 34-41 (2001)

21. Julier, S.J., Uhlmann, J.K.: A new extension of the Kalman filter to nonlinear systems. In: Proc. of AeroSense: The 11th Int. Symp. on Aerospace/Defence Sensing, Simulation and Controls., pp. 182-193 (1997)

22. Kalman, R.E.: A new approach to linear filtering and prediction problem. Trans. ASME 82, 3445 (1960)

23. Kazimierski, W., Stateczny, A.: Radar and automatic identification system track fusion in an electronic chart display and information system. J. Navig. 68(68), 1141-1154 (2015)

24. Khaleghi, B., Khamis, A., Karray, F.O., Razavi, S.N.: Multisensor data fusion: a review of the state-of-the-art. Inf. Fusion 14(1), 28-44 (2013). https://doi.org/10.1016/j.inffus.2011.08.001

25. Kim, C., Li, F., Ciptadi, A., Rehg, J.M.: Multiple hypothesis tracking revisited. In: 2015 IEEE International Conference on Computer Vision (ICCV), pp. 4696-4704 (2015). https://doi. org/10.1109/ICCV.2015.533

26. Levander, O.: Autonomous ships on the high seas. IEEE Spectr. 54(2), 26-31 (2017). https://doi.org/10.1109/MSPEC.2017.78335 02

27. Lutz, R.K.: An algorithm for the real time analysis of digitised images. Comput. J. 23(3), 262 (1980). https://doi.org/10.1093/ comjn1/23.3.262

28. Mahler, R.: A brief survey of advances in random-set fusion. In: 2015 International Conference on Control, Automation and Information Sciences (ICCAIS), pp. 62-67 (2015). https://doi. org/10.1109/ICCAIS.2015.7338726

29. Maresca, S., Braca, P., Horstmann, J.: Detection, tracking and fusion of multiple HFSW radars for ship traffic surveillance: experimental performance assessment. In: 2013 IEEE International Geoscience and Remote Sensing SymposiumIGARSS, pp. 2420-2423 (2013). https://doi.org/10.1109/IGARS S.2013.6723308

30. Mazzarella, F., Vespe, M.: SAR ship detection and self-reporting data fusion based on traffic knowledge. IEEE Geosci. Remote Sens. Lett. 12, 1685 (2015)
31. Nikolić, D., Popović, Z., Borenović, M., Stojković, N., Orlić, V., Dzvonkovskaya, A., Todorović, B.M.: Multi-radar multi-target tracking algorithm for maritime surveillance at oth distances. In: 2016 17th International Radar Symposium (IRS), pp. 1-6 (2016). https://doi.org/10.1109/IRS.2016.7497299

32. Osekowska, E., Johnson, H., Carlsson, B.: Grid size optimization for potential field based maritime anomaly detection. Transp. Res. Procedia 3, 720-729 (2014). https://doi.org/10.1016/j.trpro .2014.10.051

33. Pallotta, G., Vespe, M., Bryan, K.: Vessel pattern knowledge discovery from ais data: a framework for anomaly detection and route prediction. Entropy 15(6), 2218-2245 (2013). https://doi. org/10.3390/e15062218

34. Pulford, G.W.: Taxonomy of multiple target tracking methods. IEEE Proc. Radar Sonar Navig. 152(5), 291-304 (2005). https:// doi.org/10.1049/ip-rsn:20045064

35. Schuhmacher, D., Vo, B.T., Vo, B.N.: On performance evaluation of multi-object filters. In: 2008 11th International Conference on Information Fusion, pp. 1-8 (2008)

36. Schuster, M., Blaich, M., Reuter, J.: Collision avoidance for vessels using a low-cost radar sensor. The International Federation of Automatic Control, pp. 9673-9678 (2014)

37. Siegert, G., Banyś, P., Heymann, F.: Improving the maritime traffic situation assessment for a single target in a multisensor environment. In: Maritime Knowledge Discovery and Anomaly Detection Workshop Proceedings, European Commission-Joint Research Center, Ispra, pp. 78-82 (2016). https://doi.org/10.2788/025881

38. Siegert, G., Banyś, P., Martínez, C.S., Heymann, F.: EKF based trajectory tracking and integrity monitoring of AIS data. IEEE/ ION Position. Location and Navigation Symposium-PLANS, pp. 887-897. IEEE, Savannah (2016)

39. Siegert, G., Banyś, P., Hoth, J., Heymann, F.: Counteracting the effects of GNSS jamming in a maritime multi-target scenario by fusing AIS with radar data. In: ION International Technical Meeting, International Organization of Navigation, Monterrey (2017)

40. Tugnait, J.K.: Tracking of multiple maneuvering targets in clutter using multiple sensors, IMM and JPDA coupled filtering. In: Proceedings of the 2003 American Control Conference, vol. 2, pp. 1248-1253 (2003). https://doi.org/10.1109/ACC.2003.1239759

41. United Nations Conference on Trade and Development (UNCTAD) Review of Maritime Transport 2016, United Nations publication, chap 2, p. 5 (2016)

42. Yan, J., Liu, H., Pu, W., Jiu, B., Liu, Z., Bao, Z.: Benefit analysis of data fusion for target tracking in multiple radar system. IEEE Sens. J. 16(16), 6359-6366 (2016). https://doi.org/10.1109/ JSEN.2016.2581824 